\title{
RESPONSABILIDAD SOCIAL EMPRESARIAL COMO DETERMINANTE DE LA INTENCIÓN DE COMPRA DEL CONSUMIDOR: UN ANÁLISIS MEDIANTE MODELAMIENTO CON ECUACIONES ESTRUCTURALES ${ }^{1}$
}

CORPORATE SOCIAL RESPONSIBILITY AS A DETERMINANT OF THE CONSUMER'S PURCHASE INTENTION: AN ANALYSIS THROUGH MODELING WITH STRUCTURAL EQUATIONS

\section{RESPONSABILIDADE SOCIAL CORPORATIVA COMO DETERMINANTE DA INTENÇÃO DE COMPRA DO CONSUMIDOR: UMA ANÁLISE ATRAVÉS DA MODELAGEM COM EQUAÇÕES ESTRUTURAIS}

ENCISO ALFARO_Saudi Yulieth; RUIZ ACOSTA_Liliana Elizabeth; CAMARGO MAYORGA_ David Andrés

Contador Público, Universidad Militar Nueva Granada. Auxiliar de investigación adscrita al proyecto de investigación INV-ECO- 2969, Universidad Militar Nueva Granada. E-mail: u2303316@unimilitar.edu.co, Colombia.

Magíster en Gestión Empresarial, Universidad Libre. Docente de tiempo completo, Universidad Militar Nueva Granada. E-mail: liliana.ruiz@unimilitar.edu.co, Colombia.

Magíster en Economía, Pontificia Universidad Javeriana. Magister en Educación, Universidad Militar Nueva Granada. Docente de tiempo completo, Universidad Militar Nueva Granada. Email: david.camargo@unimilitar.edu.co, Colombia.

Recibido: 4 de junio de 2020

Aprobado: 5 de agosto de 2020

DOI: https://doi.org/10.22267/rtend.202102.138

\footnotetext{
${ }^{1}$ Artículo producto del proyecto de investigación INV-ECO 2969 "Un indicador dinámico de responsabilidad social basado en reportes GRI", financiado por la Vicerrectoría de Investigaciones de la Universidad Militar Nueva Granada en vigencia 2019.
} 


\title{
RESUMEN
}

Los consumidores están cambiando sus preferencias y prioridades de compra en atención a la implementación de prácticas de Responsabilidad Social Empresarial (RSE) vistas desde las dimensiones social, ambiental y económica, buscando minimizar el impacto negativo que las organizaciones pueden generar en su entorno. De ahí que, el objetivo de este artículo sea analizar la relación existente entre las tres dimensiones de la RSE y su influencia en la intención de compra de los consumidores. Para tal fin, se realizaron 107 encuestas en línea auto-administradas, de las cuales se obtuvieron 20 variables observables, 3 variables latentes endógenas y 1 exógena. La modelación se hizo con ecuaciones estructurales (SEM por sus siglas en inglés). Los hallazgos sugieren que las actividades de RSE que hace una organización y que están relacionadas con asuntos sociales, ambientales y económicos se asocian positivamente con la intención de compra, sin embargo, se encontró la relación con la dimensión económica no es tan fuerte como con las dos primeras.

Palabras clave: intención de compra; consumidor; sostenibilidad; responsabilidad social empresarial; modelos multivariados.

JEL: C39; C51; D12; M14; Q56

\begin{abstract}
Consumers are changing their preferences and purchasing priorities in attention to the implementation of Corporate Social Responsibility (CSR) practices seen from the social, environmental and economic dimensions, seeking to minimize the negative impact that organizations can generate in their environment. Hence, the objective of this article is to analyze the relationship between the three dimensions of CSR and its influence on the purchase intention of consumers. For this purpose, 107 self-administered online surveys were conducted, from which 20 observable variables, 3 endogenous latent variables and 1 exogenous one were obtained. The modeling was done with structural equations (SEM). The findings suggest that the CSR activities carried out by an organization and that are related to social, environmental and economic issues are positively associated with the intention to purchase, however, the relationship with the economic dimension was found not as strong as with the first two.
\end{abstract}

Keywords: purchase intention; consumer; sustainability; corporate social responsibility; multivariate models.

JEL: C39; C51; D12; M14; Q56 


\section{RESUMO}

Os consumidores estão mudando suas preferências e prioridades de compra, atendendo à implementação das práticas de Responsabilidade Social Corporativa (RSE) vistas das dimensões social, ambiental e econômica, buscando minimizar o impacto negativo que as organizações podem gerar em seu ambiente. Portanto, o objetivo deste artigo é analisar a relação entre as três dimensões da RSE e sua influência na intenção de compra dos consumidores. Para tanto, foram realizadas 107 pesquisas on-line autoaplicáveis, das quais foram obtidas 20 variáveis observáveis, 3 variáveis latentes endógenas e 1 exógena. A modelagem foi realizada com equações estruturais (MEV). Os resultados sugerem que as atividades de RSE realizadas por uma organização e relacionadas a questões sociais, ambientais e econômicas estão associadas positivamente à intenção de compra; no entanto, a relação com a dimensão econômica não foi tão forte quanto com a Os dois primeiros.

Palavras-chave: intenção de compra; consumidor; sustentabilidade; responsabilidade social empresarial; modelos multivariados.

JEL: C39; C51; D12; M14; Q56

\section{INTRODUCCIÓN}

La Responsabilidad Social Empresarial (RSE) es un tema que genera interés entre académicos y empresarios, especialmente su posible impacto en la intención de compra de los consumidores (Pérez y Topa, 2018; Yeo, Lee y Carter, 2018). La RSE implica la implementación de prácticas comerciales que contribuyan de manera positiva a la sociedad (Boronat y Pérez, 2019) a través de un proceso de cambio y participación comunitaria, con aspectos sociales, económicos y ambientales como pilares esenciales (Calderón, Cassettari, Rutkowski y de Lima Isaac, 2019).

Los consumidores principalmente de países desarrollados muestran una atención importante a las prácticas de RSE que desempeñan las organizaciones, tal y como lo establecieron Yeo et al. (2018) y Alvarado, Bigne, Aldas y Curras (2017), quienes observaron que los consumidores están cambiando sus preferencias y prioridades de compra en atención a la implementación de prácticas organizacionales que vayan en pro del bienestar general de las comunidades vistas desde una triple perspectiva: social, ambiental y económica. En contraste, las empresas han venido adoptando la RSE como una estrategia que les permita tener una fuerte relación en el largo plazo con sus 
clientes, de ello se establece la importancia de involucrar a los consumidores y a las empresas en la búsqueda del desarrollo sostenible de tal manera que le permita a los involucrados conseguir una relación óptima en pro de su entorno (Yeo et al., 2018).

Los estudios realizados sobre la relación entre la RSE desde las dimensiones social, ambiental y económica, y la intención de compra sugieren una relación positiva y significativa entre dichos constructos (Yeo et al., 2018; Xiao, Heo y Lee, 2017; Abdeen, Rajah y Gaur, 2016).

El objetivo de este artículo es analizar la relación entre las tres dimensiones de la RSE (económica, social y ambiental) y su influencia en la intención de compra de los consumidores. Este trabajo se basó en el estudio realizado por Yeo et al. (2018) en Malasia acerca de la influencia de los constructos adoptados de Responsabilidad Social Empresarial por parte de las organizaciones y la consideración de las mismas por parte de los consumidores antes de realizar cualquier compra.

El documento se encuentra estructurado así: inicialmente se encuentra el contenido introductorio, seguido de una descripción de las dimensiones de la RSE desde el desarrollo sostenible y su relación con la intención de compra. En la tercera parte el documento contiene el tipo de metodología aplicada, seguida de los resultados y discusión generados en la investigación, y por último, se presentan unas conclusiones.

\section{REFERENTES TEÓRICOS}

\section{RSE y desarrollo sostenible}

Según Alvarado et al. (2017) el concepto de Responsabilidad Social Empresarial es considerado como amplio y variado, estas concepciones abarcan ideas tales como, la obligación legal que tienen las empresas de contribuir al mejoramiento continuo del nivel de vida de la comunidad en general llevando a cabo actividades tales como el desarrollo de programas de capacitación, atención médica y oportunidades educativas (Mohd, Ismail y Alias, 2015; Smith, 2013). Por otro lado, Daza (2009) indica que la RSE es el intento que hace una empresa por cumplir sus tareas de forma extraordinaria para seguir existiendo y asegurar su supervivencia. Según este autor, esto requiere que una empresa cumpla ciertas condiciones como actuar más allá del mínimo regulatorio, es decir llevar a cabo actividades voluntarias (Yeo et al., 2018), actuar con ética en todas las instancias de toma de decisiones que son inseparables de la cultura de la compañía, brindar condiciones de 
trabajo seguras y saludables, respetar el medio ambiente, e integrar la empresa en la comunidad en la que se inserta.

Adicionalmente, Gonçalves das Neves, Mouro y Duarte (2010) establecen que la RSE se relaciona con las cuestiones filosóficas y normativas del papel que juega la empresa en la sociedad. Los supuestos básicos de la RSE según estos autores están estrechamente relacionados con los principios del desarrollo sostenible, a saber, que las corporaciones deben tomar decisiones basadas no sólo en factores financieros y económicos (por ejemplo, ganancias, rendimiento de la inversión, pagos de dividendos y otros), sino también sociales (condiciones de trabajo, capacitación, bienestar de la comunidad general, entre otros) y medioambientales (cuidado y preservación de los ecosistemas). En otras palabras, “exige una convergencia entre el desarrollo económico, la equidad social y la protección del medio ambiente" (Alvarado et al., 2017: 245) con el fin de satisfacer las necesidades del presente sin comprometer la capacidad de las generaciones futuras para satisfacer sus propias necesidades (Brundtland, Khalid, Agnelli y Al-Athel, 1987).

Aunado a lo anterior, Alvarado et al. (2017) aseguran que el término de desarrollo sostenible se ha descuidado a pesar de que ofrece una perspectiva útil para conceptualizar la RSE, debido a que se convierte en una híper norma que ofrece principios universales exigiendo atención a nivel organizacional para convertir la perspectiva tradicional de la rentabilidad económica en un triple resultado (económico, social y medioambiental) y de esa forma generar acciones de RSE que sean amplias, tangibles y fáciles de evaluar por parte de los consumidores (ver Figura 1).

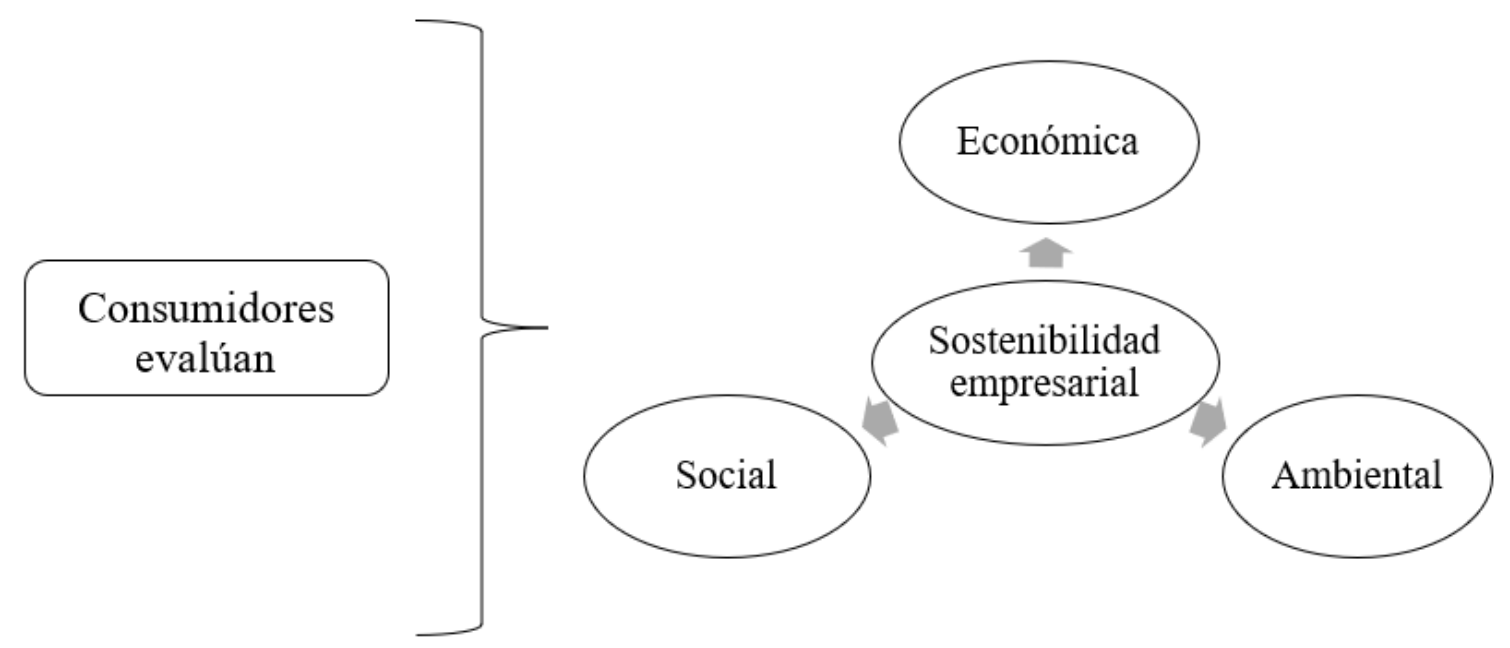

Figura 1. Consumidores y Triple resultado.

Fuente: elaboración propia a partir de Elkington (1994) y Alvarado et al. (2017). 
De ahí que, estos autores aseguren que una definición apropiada para la RSE es "el compromiso que tiene una empresa por maximizar (...) el bienestar económico, social y ambiental a largo plazo a través de prácticas y políticas de negocio" (245) compatibles con su entorno. Además, afirman que esta definición es apropiada, porque permite la identificación de las dimensiones de la RSE directamente desde el modelo de desarrollo sostenible y garantiza la operatividad de la percepción de los consumidores sobre las prácticas comerciales y políticas organizacionales de RSE, apoyando la idea de que la RSE es un constructo reflexivo tridimensional (Alvarado et al., 2017).

\section{RSE e intención de compra de los consumidores}

Abdeen et al. (2016) establecen que el comportamiento social humano se produce espontáneamente a partir de sus creencias, percepciones y de la evaluación de las consecuencias de sus actos. Así, las creencias forman la base de la cual emergen las actitudes, las intenciones y comportamientos. Ferraz, Buhamra, Laroche y Veloso (2017) exponen que las intenciones actúan como un indicador de la medida en que una persona estaría dispuesta a comprar. La medición de las intenciones de compra a menudo se ha utilizado para identificar la potencialidad de venta de los productos porque cuanto mayor es la intención, mayor será la probabilidad de compra.

Como lo explican Kong y Chen (2009); Gupta (2013); Pérez y Topa (2018); y Golob y Kronegger (2019), las expectativas de los consumidores por las acciones de RSE que llevan a cabo las compañías se hacen cada vez más fuertes, los consumidores esperan que las empresas participen en iniciativas que representen un bienestar equilibrado entre las dimensiones económica, social y medioambiental de la RSE, de tal forma que dichas expectativas se han convertido en un componente importante en el proceso de tomar decisiones de compra por parte de los consumidores, es decir como lo establecen Tuan, Dalli, Gandolfo y Gravina (2019) y Wang y Lee, (2018) la RSE se ha convertido en una manera de influir en la forma en que los consumidores denotan a las compañías y sus productos. Pérez y Topa (2018) y Abdeen et al. (2016) determinaron que la información positiva de la RSE sobre una empresa mejora las intenciones de los consumidores de comprar productos, además aseguran que es vital que las empresas seleccionen y apliquen iniciativas de RSE relevantes y congruentes para garantizar resultados exitosos.

Yeo et al. (2018) plantearon inicialmente que la intención de compra de los consumidores, en la mayoría de casos, podía llegar a depender significativamente de las características demográficas, como la edad, el género, el nivel de ingresos y la educación. Sin embargo, los autores establecieron 
que "en términos del impacto de los componentes demográficos en el comportamiento del consumidor, los resultados representaron una relación e influencia insignificantes entre todas las características de la muestra evaluada" (Yeo et al., 2018: 462) para el caso de Malasia. Teniendo en cuenta esta situación, en este trabajo se busca establecer la relación que existen entre las tres dimensiones de RSE y la intención de compra de los consumidores.

\section{RSE -dimensión social e intención de compra}

“Además de obtener ganancias, una organización debe tener en cuenta las implicaciones sociales de su actividad y tratar de maximizar y contribuir con un impacto positivo para ellas" (Yeo et al., 2018: 452). Dada la velocidad actual con la que pueden propagarse las noticias se hace difícil para las compañías ignorar o encubrir las violaciones de los derechos humanos y la agitación social relacionada con sus actividades (McLennan y Banks, 2019), por ello las empresas buscan mitigar dichos impactos negativos mediante la implementación de prácticas de RSE, como son la generación de códigos de conducta (...) para garantizar los derechos humanos y las condiciones de los trabajadores; implementando acuerdos de comercio justo; brindando programas educativos o de atención médica para garantizar el bienestar de la comunidad (McCarthy, Claudy, Marshall y McGrath, 2015); realizando campañas de seguridad y salud dirigidas a los consumidores (Plasencia, Marrero, Bajo y Nicado, 2018).

Galuppo, Gorli, Scaratti y Kaneklin (2014) indican que la dimensión social del desarrollo sostenible pretende ser el dominio sinérgico y estratégico para los dominios económico y ambiental. Por lo tanto, la producción de valor social representa un activo clave para enriquecer a las otras dos dimensiones, lo que lleva a que una organización "devuelva a la sociedad en lugar de simplemente explotar los recursos disponibles" (Docherty, Kira, y Shani, 2008: 5). Según explican Joo, Miller y Fink (2019) los consumidores perciben como reales las iniciativas de RSE cuando benefician de manera directa y tangible a su comunidad generando un considerable impacto en la intención de compra de los consumidores. Lo anterior llevó al planteamiento de la primera hipótesis:

H1: La dimensión social de la RSE es un factor que determina la intención de compra de los consumidores. 


\section{RSE -dimensión ambiental e intención de compra}

Plasencia et al. (2018) establecen que la base de la dimensión medioambiental es el cuidado y protección de los recursos naturales no renovables y de la biodiversidad, mediante la reducción de emisiones de gases de efecto invernadero, programas de procesamiento de desechos, entre otros. Yeo et al. (2018) aseguran que las compañías buscan obtener buenos créditos a los ojos de los consumidores, con la divulgación del cuidado medioambiental mediante la comunicación de forma honesta y transparente de errores que, la compañía cometa en menoscabo de los ecosistemas o brindando educación a los consumidores sobre cómo pueden mejorar el medio ambiente y hacer una diferencia en su comunidad, con el fin de tener un aumento en sus ventas.

En contraste con lo anterior, Golob y Kronegger (2019) afirmaron que algunos consumidores buscan conscientemente minimizar el impacto negativo de las prácticas empresariales que degradan el medioambiente, debido a la creciente preocupación por el calentamiento global, el cambio climático y por un medio ambiente saludable para vivir (Agarwal y Kasliwal, 2017), por ello deciden adquirir bienes o servicios de empresas que presten principal atención al diseño de sistemas productivos que sean capaces de usar únicamente recursos y energías renovables, y en lo posible no producir residuos o tratarlos de forma adecuada (Artaraz, 2002), también esperan que las organizaciones protejan las fuentes hídricas, la biodiversidad y hábitats naturales, entre otros" (Plasencia et al., 2018). Por último, Yang (2017) demostró que un número creciente de consumidores prefiere comprar productos con etiquetas ecológicas que indiquen un menor impacto ambiental de las operaciones corporativas, puesto que de este modo los consumidores se pueden integrar con las compañías para generar cambios en beneficio de su entorno. De lo anterior surgió el planteamiento de la segunda hipótesis:

H2: La dimensión ambiental de la RSE es un factor que determina la intención de compra de los consumidores.

\section{RSE -dimensión económica e intención de compra}

La dimensión económica “es la unidad fundamental de una compañía en la que una empresa debe garantizar condiciones económicas sostenibles y condiciones financieras estables para el éxito comercial continuo" (Yeo et al., 2018: 451), el cual "incluye aspectos tales como la producción, los costos" (Plasencia et al., 2018: 65) “el crecimiento del negocio, la equidad en los precios donde 
los clientes perciben que el precio de un bien o servicio vale la pena en términos de calidad y beneficios" (Yeo et al., 2018: 451).

Según el estudio de Xiao et al. (2017) los consumidores perciben la dimensión económica como menos importante en comparación con las otras dos dimensiones, dándose una relación negativa entre dicha dimensión y la intención de compra o apoyo que los consumidores tienen hacia las empresas que realizan actividades en ese sentido. Sin embargo, los autores afirman que este resultado puede ser diferente en otros contextos culturales debido principalmente a la forma en cómo los consumidores perciben las crisis económicas y sus consecuencias como lo son el desempleo, la inseguridad y los riesgos financieros. Lo anterior llevó al planteamiento de la tercera hipótesis:

H3: La dimensión económica de la RSE es un factor que determina la intención de compra de los consumidores.

\section{METODOLOGÍA}

Para el presente estudio se utilizó un enfoque cuantitativo, en el que se realizó una encuesta online auto-administrada con el fin de analizar la relación entre las tres dimensiones de la RSE (social, económica y ambiental) y su influencia en la intención de compra de los consumidores en general.

\section{Diseño de la encuesta}

Las afirmaciones para las encuestas se adaptaron de la escala de medición para las percepciones de los consumidores de la Responsabilidad Social Corporativa (CSRConsPerScale) desarrollada por Alvarado et al. (2017), y las afirmaciones propuestas por Yeo et al. (2018).

El cuestionario estuvo compuesto por dos secciones, en la primera parte se buscó conocer los datos demográficos de los encuestados, como edad, género, nivel de ingresos y de educación. La segunda sección contenía afirmaciones referidas a la intención de compra de los consumidores en relación con las prácticas de RSE enmarcadas en las dimensiones del desarrollo sostenible. Las tres dimensiones de la RSE se consideraron como variables latentes y las afirmaciones como variables observables. 
La sección dos contó con una escala tipo Likert de cinco puntos (1-muy en desacuerdo, 2-en desacuerdo, 3-ni de acuerdo ni en desacuerdo, 4-de acuerdo, 5-muy de acuerdo) para valorar la importancia de las afirmaciones que relacionan la RSE desde las dimensiones del desarrollo sostenible y la intención de compra de los consumidores. El instrumento se probó a través de una prueba piloto en un grupo de seis consumidores antes de la divulgación de la encuesta vía correo electrónico y redes sociales de mensajería instantánea. Una vez hecha la prueba piloto, se realizaron modificaciones menores a las afirmaciones con el fin de asegurar que estuvieran libres de ambigüedad o falta de comprensión.

\section{Muestra y método}

Según lo establecido por Roscoe (1975) los tamaños de muestra mayores de 30 y menores de 500 son apropiados para la mayoría de los estudios, por su parte, Ruiz, Pardo y San Martín (2010) establecen que en los modelos de ecuaciones estructurales se acostumbra a exigir tamaños muéstrales superiores a los 100 sujetos debido a que constituye una buena garantía para el desarrollo de cualquier investigación que emplee dichos modelos. En trabajos relacionados con responsabilidad social los tamaños de muestras menores a 500 se han usado por Mustafa, Othman y Perumal, (2012), Rahim, Jalaludin y Tajuddin, (2011), entre otros. De esta forma la investigación se dirigió a un tamaño de muestra de 115 consumidores en Bogotá, de los cuales se obtuvieron 107 respuestas válidas y utilizables.

Para analizar las relaciones existentes entre las dimensiones de RSE (variables latentes) y las afirmaciones (variables observables) y la intención de compra de los consumidores se utilizó el modelo de ecuaciones estructurales SEM (ver Figura 2). El SEM es un modelo multivariado que representa relaciones entre variables observadas y no observadas para cuantificar si las hipótesis estipuladas por el investigador son compatibles permitiendo combinar y confrontar la teoría con los datos obtenidos (Ashman y Patterson, 2015), para ello es necesario "utilizar un conjunto de ecuaciones que representen las relaciones propuestas por la teoría” (Ruiz et al., 2010: 35). 


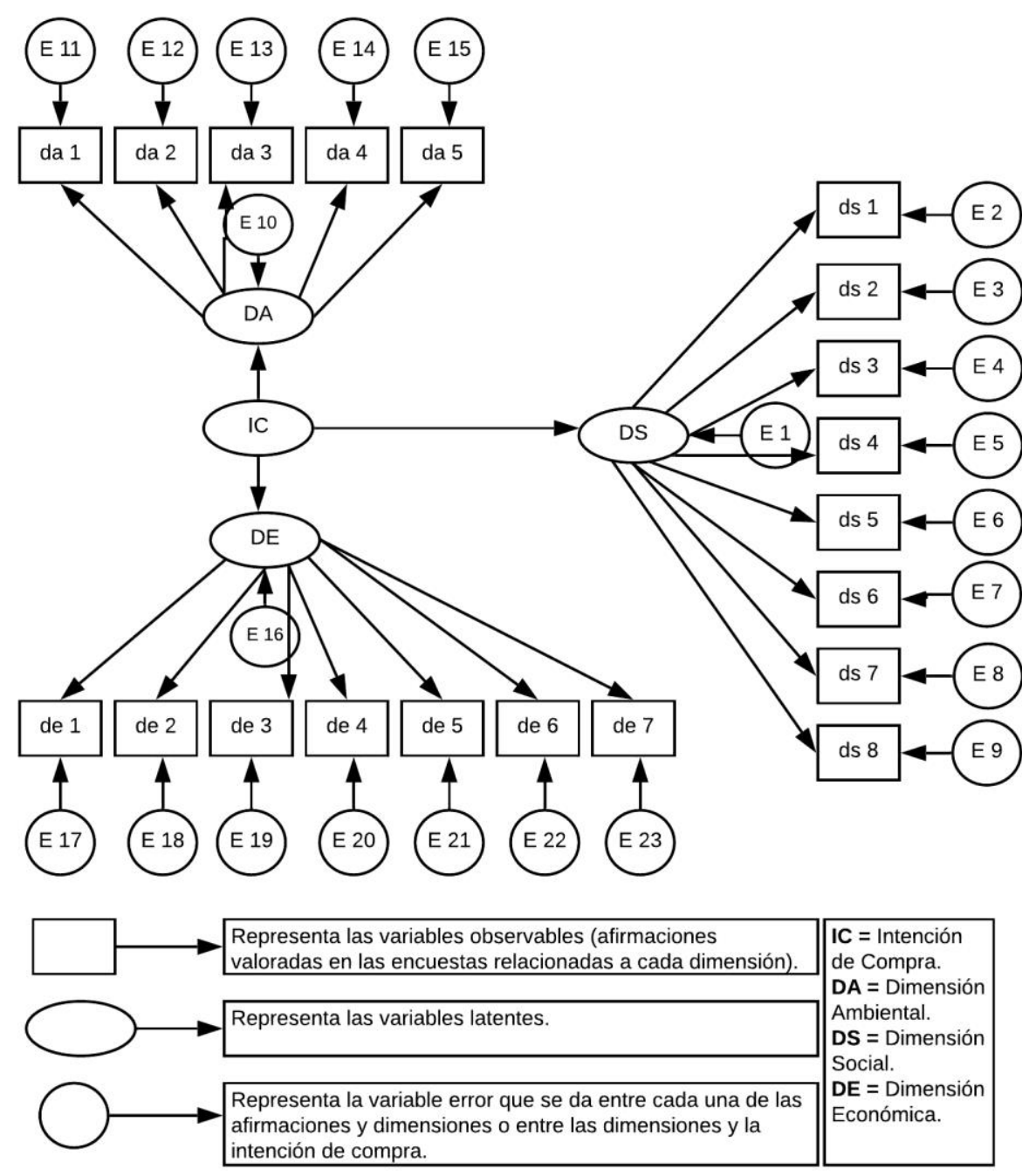

Figura 2. Modelo teórico.

Fuente: elaboración propia a partir de Alvarado et al. (2017) y Yeo et al. (2018).

En estos modelos se distinguen distintos tipos de variables dependiendo de su papel o medición, para el caso del presente estudio se tiene que una variable latente corresponde a la "característica que se desearía medir pero que no se puede observar y que está libre de error de medición” (Ruiz et al., 2010: 36). En cuanto a la variable observable "es la que mide a los sujetos. Por ejemplo, las preguntas de un cuestionario" (Ruiz et al., 2010: 36). Por último, se tiene la variable error que "representa tanto los errores asociados a la medición de una variable como el conjunto de variables que no han sido contempladas en el modelo y que pueden afectar a la medición de una variable observada" (Ruiz et al., 2010: 36). Los datos recolectados se analizaron utilizando el software estadístico Stata 14. 


\section{RESULTADOS Y DISCUSIÓN}

De acuerdo con el total de respuestas válidas obtenidas (107), la participación por género fue del $55 \%$ para las mujeres y el $45 \%$ para los hombres, la edad promedio de los participantes estuvo alrededor de los 29 años lo cual indica que los encuestados tenían su propio poder de compra. El grupo más representativo de los encuestados en cuanto al nivel educativo fue el de pregrado con el 50\% de participación. En cuanto al nivel de ingresos los resultados indicaron que el 34\% de los consumidores contaban con un ingreso mensual promedio mayor a $\$ 830.000$ y menor a $\$ 1.660 .000$ (pesos colombianos) siendo el grupo con mayor participación.

En cuanto a la intención de compra relacionada con el perfil demográfico de los encuestados no se tuvo en cuenta para el análisis de la presente investigación debido a la relación poco significativa encontrada por Yeo et al. (2018) entre dichas variables.

En lo que respecta a la fiabilidad del cuestionario, se realizó el análisis de fiabilidad mediante un índice de consistencia interna, el estadístico alfa de Cronbach para cada una de las afirmaciones. Según Yeo et al. (2018) el coeficiente alfa oscila entre 0 y 1, donde 0 significa que el cuestionario no es consistente y 1 que el cuestionario tiene una consistencia perfecta. En esta investigación, se obtuvo un alfa de Cronbach con un valor superior a 0.96 para todas las afirmaciones, lo cual indica que las escalas de medición de todos los constructos eran confiables en términos de medir sus respectivos constructos.

Como lo establecieron Alvarado et al. (2017) la valoración por parte de los consumidores de la RSE es una construcción compleja que se refleja en la discriminación de las dimensiones económica, social y ambiental. Para examinar individualmente la relación entre las tres dimensiones de la RSE y el comportamiento de compra del consumidor se utilizó el modelo SEM, cuyos resultados se muestran en la Figura 3. Los índices de ajuste del modelo son aceptables con un CFI de 0.86 y un RMSEA de 0.135 (p-close menor del 5\%). 


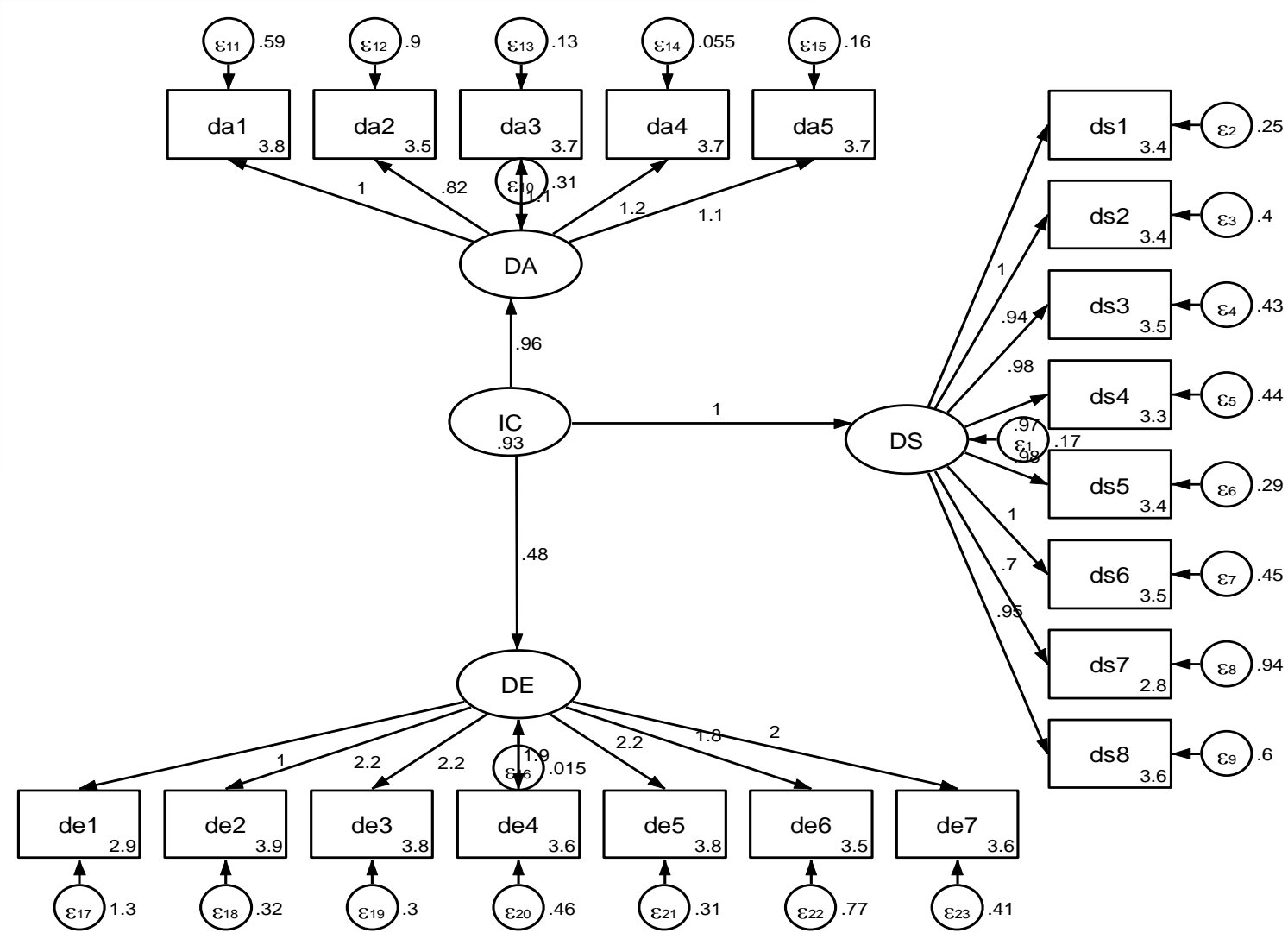

Figura 3. Modelo SEM con resultados.

Fuente: elaboración propia en Stata 14.

Los resultados de la Tabla 1 indican que la RSE desde la dimensión social (L1) se asocia positivamente con la intención de compra (IC) del consumidor ( $\mathrm{r}=1$ ). La IC se asoció fuertemente con el apoyo económico a programas educativos y/o culturales y con la ayuda para mejorar la calidad de vida de la comunidad local que una organización puede brindar. Es decir, los consumidores perciben como reales las iniciativas de RSE que impactan de forma directa y positiva a la comunidad en la que se insertan (Joo et al., 2019).

\section{Tabla 1}

Relación de las tres dimensiones de la RSE y la intención de compra

\begin{tabular}{lllll}
\hline Relación & Estimado & $\mathrm{P}$ & Hipótesis & Cumplimiento \\
\hline L1 $\rightarrow$ IC & 1 & -- & H1 & Acepta \\
L2 $\rightarrow$ IC & 0.9576 & 0.000 & H2 & Acepta \\
L3 $\rightarrow$ IC & 0.4812 & 0.000 & H3 & Acepta
\end{tabular}

Fuente: elaboración propia. 
Además, cabe resaltar que las actividades de RSE enmarcadas en la dimensión social son las más importantes para los consumidores en relación con las otras dos dimensiones, puesto que en la actualidad como lo indican Docherty et al. (2008) los consumidores no están dispuestos a ser vistos por las empresas como el mercado potencial por medio del cual pueden obtener los recursos para crecer o enriquecerse, sino como un grupo de personas capaces de emprender acciones en pro del bienestar de sus comunidades locales.

En cuanto a la dimensión ambiental de la RSE (L2, la Tabla 1 muestra que se asoció de forma positiva con la intención de compra (IC) del consumidor (r=0,957). Como lo explican Yeo et al. (2018) en los últimos años se ha venido presentando un patrón de comportamiento por parte de los consumidores que indica que ellos han comenzado a darse cuenta de lo importante que es la propuesta de valor de la RSE para el medioambiente por lo que brindan un apoyo importante a empresas que emprendan acciones en pro de los ecosistemas tal y como lo indican los resultados del presente trabajo.

Adicionalmente, aunque la dimensión ambiental de la RSE se encuentra en un segundo lugar de importancia para los consumidores respecto de las otras dimensiones, no deja de tener una relación fuerte con la intención de compra. Los consumidores están de acuerdo con su responsabilidad personal y su capacidad para desempeñar un papel activo en la sociedad relacionado con la protección ambiental, llevándolos a estar dispuestos a premiar con sus compras a las empresas que cumplan con sus expectativas, aunque ello implique asumir algunos costos personales, como el pago de precios más altos (Golob y Kronegger, 2019; Yang, 2017).

Por otro lado, la dimensión económica (L3) como se puede observar en la Tabla 1, aunque se asoció de forma positiva con la intención de compra, su relación no es fuerte como en el caso de las otras dos dimensiones ( $\mathrm{r}=0,481)$, lo cual es acorde con lo encontrado por Xiao et al. (2017) y como los autores lo plantearon la poca importancia dada por los consumidores a las actividades que una organización realiza enmarcadas en dicha dimensión, "puede deberse a una falta de comprensión clara de la dimensión económica, que en el contexto de la RSE, debe implicar una perspectiva a largo plazo que brinde a las empresas sostenibilidad económica (...). Sin embargo, el marco contemporáneo de la dimensión económica de la RSE puede necesitar un mayor y continuo desarrollo para reflejar el entorno siempre cambiante del mundo corporativo" (704) y sus efectos en la sociedad. 
Lo expuesto en este punto permite establecer que las hipótesis planteadas (H1, H2 y H3) fueron aceptadas teniendo en cuenta las implicaciones descritas en los párrafos anteriores, es decir que las actividades de RSE que puede llegar a emprender una organización en el contexto de las tres dimensiones del desarrollo sostenible influyen de forma positiva en la intención de compra de los consumidores (ver Tabla 1).

\section{CONCLUSIONES}

En términos generales es significativa la incidencia de las prácticas de RSE en la intención de compra de los consumidores como se evidenció en los resultados. De ello, es posible concluir que las empresas deben atender las demandas de los consumidores, yendo más allá de la simple venta, para impulsar con sus acciones un equilibrio tridimensional de los aspectos de la RSE. Esto puede llegar a garantizar su sostenibilidad en un contexto cada vez más exigente y que implica prácticas basadas en lo social, lo ambiental y lo económico.

Como se evidenció, los consumidores no establecen una fuerte relación con la dimensión económica de la RSE, posiblemente porque desconocen esta, como es sugerido por la literatura al respecto. Es importante que las empresas den a conocer la profundidad de la dimensión económica en el contexto de la RSE, para que así los consumidores no solo piensen que las organizaciones deben contribuir a un bienestar social con apoyos económicos a las personas menos favorecidas. Sino que además, piensen en el compromiso social que estas tienen cuando son estables financieramente y benefician con su existencia a empleados, proveedores, accionistas, entre otros grupos de interés.

La mayoría de los estudios que se han realizado acerca de la RSE y la relación con los consumidores se han gestado en países desarrollados, de donde surge la necesidad de realizar más trabajos que investiguen las relaciones existentes entre la RSE, el desarrollo sostenible, y las percepciones y reacciones de los consumidores ante la implementación de prácticas socialmente responsables en el contexto colombiano y latinoamericano, con el fin de validar constructos que den cuenta de esa realidad.

Dado que los consumidores son fundamentales para el cambio hacia una sociedad sostenible, la sofisticación de la demanda como mecanismo que impulse un consumidor responsable, genera 
también la necesidad de desarrollar líneas de investigación en RSE que involucren aspectos relacionados con la formación de ciudadanos que tengan estilos de vida sostenible referidos a la comprensión de la huella ecológica, el uso de energía, la movilidad, la economía circular y las finanzas verdes, entre otros temas fundamentales que permitirán al ser humano vivir en armonía con el planeta y con los otros seres vivos.

Por último, es pertinente que las organizaciones hagan la identificación de aquellas actividades de RSE que influyen de forma directa y significativa en la intención de compra de los consumidores, teniendo en cuenta su entorno empresarial, con el fin de potenciarlas a través del direccionamiento estratégico, generar bienestar equilibrado y consolidarse como empresas que perduren.

\section{REFERENCIAS}

(1) Abdeen, A., Rajah, E., y Gaur, S. (2016). Consumers' beliefs about firm's CSR initiatives and their purchase behaviour. Marketing Intelligence and Planning, 34(1), 2-18. doi: 10.1108/MIP-06-20140107

(2) Agarwal, S. y Kasliwal, N. (2017). The Effect of Age as a Moderator on Green Purchase Behavior in Hotel Industry. IPE Journal of Management, 7(2), 53-63.

(3) Alvarado, A., Bigne, E., Aldas, J. y Curras, R. (2017). A Scale for Measuring Consumer Perceptions of Corporate Social Responsibility Following the Sustainable Development Paradigm. Journal of Business Ethics, 140(2), 243-262. doi: 10.1007/s10551-015-2654-9

(4) Artaraz, M. (2002). Teoría de las tres dimensiones de desarrollo sostenible. Revista Ecosistemas, 11(2). doi: 10.7818/re.2014.11-2.00

(5) Ashman, R. y Patterson, A. (2015). Seeing the big picture in services marketing research: infographics, SEM and data visualisation. Journal of Services Marketing, 29(6/7), 613-621. doi: 10.1108/JSM-01-2015-0024

(6) Boronat, M. y Pérez, J. A. (2019). Consumers' perceived corporate social responsibility evaluation and support: The moderating role of consumer information. Tourism Economics, 25(4), 613-638. doi: $10.1177 / 1354816618812297$

(7) Brundtland, G. H., Khalid, M., Agnelli, S. y Al-Athel, S. (1987). Our common future. New York.

(8) Calderón, A. J., Cassettari, P. C., Rutkowski, E. W. y de Lima Isaac, R. (2019). Landfill mining as a strategic tool towards global sustainable development. Journal of Cleaner Production, 226, 11021115. doi: 10.1016/j.jclepro.2019.04.057 
Responsabilidad social empresarial como determinante de la intención de compra del consumidor: un análisis mediante modelamiento con ecuaciones estructurales

Enciso Alfaro_Saudi Yulieth; Ruiz Acosta_Liliana Elizabeth; Camargo Mayorga_ David Andrés

(9) Daza, R. (2009). A valuation model for corporate social responsibility. Social Responsibility Journal, 5(3), pp-284-299. doi: 10.1108/17471110910977230

(10) Docherty, P., Kira, M. y Shani, A. B. (2008). Creating Sustainable Work Systems: Developing Social Sustainability. Routledge.

(11) Elkington, J. (1994). Towards the Sustainable Corporation: Win-Win-Win Business Strategies for Sustainable Development. California Management Review, 36(2), 90-100. doi: 10.2307/41165746

(12) Ferraz, S. B., Buhamra, C., Laroche, M. y Veloso, A. R. (2017). Green Products: A Cross-Cultural Study of Attitude, Intention and Purchase Behavior. Revista de Administração Mackenzie; São Paulo, 18(5), 12-38. doi: 10.1590/1678-69712017/administracao.v18n5p12-38

(13) Galuppo, L., Gorli, M., Scaratti, G. y Kaneklin, C. (2014). Building social sustainability: multistakeholder processes and conflict management. Social Responsibility Journal, 10(4), 685-701. doi: 10.1108/SRJ-10-2012-0134

(14) Golob, U. y Kronegger, L. (2019). Environmental consciousness of European consumers: A segmentation-based study. Journal of Cleaner Production, 221, 1-9. doi: 10.1016/j.jclepro.2019.02.197

(15) Gonçalves das Neves, J., Mouro, C. y Duarte, A. (2010). Corporate social responsibility: mapping its social meaning. Management Research: Journal of the Iberoamerican Academy of Management, 8(2), 101-122. doi: 10.1108/1536-541011066461

(16) Gupta, S. (2013). Consumer stakeholder view of corporate social responsibility: a comparative analysis from USA and India. Social Responsibility Journal, 7(3), 363-380. doi: $10.1108 / 17471111111154518$

(17) Joo, S., Miller, E. G. y Fink, J. S. (2019). Consumer evaluations of CSR authenticity: Development and validation of a multidimensional CSR authenticity scale. Journal of Business Research, 98, 236249. doi: 10.1016/j.jbusres.2019.01.060

(18) Kong, Y. y Chen, H. (2009). Chinese consumer perceptions of socially responsible consumption. Social Responsibility Journal, 5(2), 144-151. doi: 10.1108/17471110910964441

(19) McCarthy, L., Claudy, M., Marshall, D. y McGrath, P. (2015). Going above and beyond: how sustainability culture and entrepreneurial orientation drive social sustainability supply chain practice adoption. Supply Chain Management, 20(4), 434-454. doi: 10.1108/SCM-08-2014-0267

(20) McLennan, S. y Banks, G. (2019). Reversing the lens: Why corporate social responsibility is not community development. Corporate Social Responsibility and Environmental Management, 26(1), 117-126. doi: https://doi.org/10.1002/csr.1664

(21) Mohd, R., Ismail, M. y Alias, S. N. (2015). Community as stakeholder of the corporate social responsibility programme in Malaysia: outcomes in community development. Social Responsibility Journal, 11(1), 109-130. doi: 10.1108/SRJ-05-2013-0053 
(22) Mustafa, S. A., Othman, A. R. y Perumal, S. (2012). Corporate Social Responsibility and Company Performance in the Malaysian Context. Procedia - Social and Behavioral Sciences, 65, 897-905. doi: 10.1016/j.sbspro.2012.11.217

(23) Pérez, S. A., y Topa, G. (2018). Percepciones sobre la responsabilidad social corporativa de las empresas: relación con las actitudes y conductas de sus clientes. Acción Psicológica; Madrid, 15(1), 103-119. doi: 10.5944/ap.15.1.22003

(24) Plasencia, J. A., Marrero, F., Bajo, A. M. y Nicado, M. (2018). Modelos para evaluar la sostenibilidad de las organizaciones. Estudios Gerenciales, 34(146), 63-73. doi: 10.18046/j.estger.2018.146.2662

(25) Rahim, R. A., Jalaludin, F. W. y Tajuddin, K. (2011). The importance of Corporate Social Responsibility on consumer behaviour in Malaysia. Asian Academy of Management Journal, 16(1), 119-139.

(26) Roscoe, J. T. (1975). Fundamental Research Statistics for the Behavioral Science. New York, The United States of America: Holt, Rinehart and Winston Inc.

(27) Ruiz, M., Pardo, A. y San Martín, R. (2010). Modelos de Ecuaciones estructurales. Papeles del Psicólogo, 31(1), 34-45.

(28) Smith, A. D. (2013). Corporate social responsibility practices in the pharmaceutical industry. Business Strategy Series, 9(6), 306-315. doi: 10.1108/17515630810923612

(29) Tuan, A., Dalli, D., Gandolfo, A. y Gravina, A. (2019). Theories and methods in CSRC research: a systematic literature review. Corporate Communications: An International Journal, 24(2), 212-231. doi: 10.1108/CCIJ-11-2017-0112

(30) Wang, C. y Lee, J. (2018). Doing Right Matters in Doing Good: The Role of CSR Fit on Building Company Credibility and Reputation Through Consumer Attributions. Journal of Leadership, Accountability and Ethics, 15(1), 11-28. doi: 10.33423/jlae.v15i1.627

(31) Xiao, Q., Heo, C. Y. y Lee, S. (2017). How do consumers' perceptions differ across dimensions of corporate social responsibility and hotel types? Journal of Travel \& Tourism Marketing, 34(5), 694707. doi: 10.1080/10548408.2016.1232671

(32) Yang, F. (2017). Integrating corporate social responsibility and profitability into best practice selection: the case of large Taiwanese firms. Quality \& Quantity, 51(4), 1493-1512. doi: 10.1007/s11135-016-0348-8

(33) Yeo, A. C.-M., Lee, S. X.-M. y Carter, S. (2018). The influence of an organisation's adopted corporate social responsibility constructs on consumers' intended buying behaviour: a Malaysian perspective. Social Responsibility Journal, 14(3), 448-468. doi: 10.1108/SRJ-05-2016-0082

Cómo citar este artículo: Enciso, S., Ruiz, L. y Camargo, D. (2020). Responsabilidad social empresarial como determinante de la intención de compra del consumidor: un análisis mediante modelamiento con ecuaciones estructurales. Tendencias, 21(2), 1-18. https://doi.org/10.22267/rtend.202102.138 